\title{
Theory overview of open heavy flavor probes of QCD matter
}

\section{Shanshan Cao*}

Department of Physics and Astronomy, Wayne State University, Detroit, MI, 48201, USA

E-mail: shanshan.cao@wayne.edu

\begin{abstract}
A brief theory overview of open heavy quarks is presented in this work. Different theories and models are summarized for heavy quark energy loss and hadronization inside the QGP. Two newly developed multi-scale evolution approaches are then introduced for heavy quark energy loss, which combine heavy quark interaction with the medium at different virtuality scales, and different scales of the momentum exchange. In the end, recent theoretical progress on probing the medium properties using heavy quarks has been presented, including probing the QGP evolution history and probing the initial state effects in high-energy nuclear collisions.
\end{abstract}

International Conference on Hard and Electromagnetic Probes of High-Energy Nuclear Collisions 30 September - 5 October 2018

Aix-Les-Bains, Savoie, France

${ }^{*}$ Speaker. 


\section{Introduction}

Heavy quarks are ideal probes of the quark-gluon plasma (QGP) matter produced in highenergy nuclear collisions. They provide a unique opportunity to study parton-medium interaction at different scales. At low transverse momenta $\left(p_{\mathrm{T}}\right)$, one observes $D$ mesons and light flavor hadrons share the similar number-of-constituent-quark-scaled elliptic flow coefficient $\left(v_{2}\right)$ [1], indicating charm quarks exhibit thermal features of the QGP. This allows us to study the thermalization process of heavy quarks and constrain their diffusion coefficient. At medium $p_{\mathrm{T}}$, the $\Lambda_{c} / D_{0}$ ratio is much larger in nucleus-nucleus (AA) collisions than in proton-proton (pp) collisions [2]. This guides the improvement of our understanding of the heavy quark hadronization process, and may also help constrain the hadron wave-function. At high $p_{\mathrm{T}}$, experimental data indicate a similar nuclear modification factor between light flavor hadrons, $D$ mesons and $B$ mesons [3], urging us to re-visit parton energy loss inside the QGP, especially its mass dependence, and constrain the jet transport coefficient.

In this work, I will first review heavy quark theories and models at different momentum scales. Then I will present several newly developed multi-scale approaches for heavy quark energy loss. In the end, I will discuss recent developments on utilizing heavy quarks to probe the QGP properties, as well as remaining puzzles in the heavy quark field.

\section{Heavy quark theories and models at different momentum scales}

At low momenta, the diffusion of heavy quarks inside a thermal medium is characterized by their momentum space diffusion coefficient $\kappa$, or the spatial diffusion coefficient $D_{\mathrm{s}}$. The leadingorder (LO) perturbative calculation of this $\kappa$ was first provided in Ref. [4] at zero momentum and then extended to finite momentum in Ref. [5]. The next-to-leading-order (NLO) calculation was given in Ref. [6], where one observes that the NLO result is larger than the LO result by a factor of 5 when the strong coupling constant $\alpha_{\mathrm{s}}$ is around 0.2 to 0.3 . This indicates the failure of the perturbative method for low momentum heavy quarks. One can also evaluate the diffusion coefficient on lattice $[7,8,9]$. However, due to the current large error bars and the limitation of results at zero momentum, we have no reliable input from lattice for model calculations to compare to realistic observables.

In reality, modeling is required to understand heavy flavor observables at low $p_{\mathrm{T}}$. A nonperturbative resonance scattering method was developed in Refs [10,11], where the authors assume heavy-light quark interaction with certain potential, and then solve the $T$-matrix, from which the diffusion coefficient can be extracted. Due to the existence of the resonant states, the energy loss is enhanced compared to the perturbative calculation, and thus the spatial diffusion coefficient $D_{\mathrm{s}}$ is suppressed. Recently, the authors break through the limitation of approximating the heavy-light quark interaction potential with either the free energy or the internal energy from the lattice QCD calculation, and manage to solve the real potential self-consistently within the $T$-matrix framework [12]. An alternative approach is constructing quasi-particle models to simulate heavy quark interaction with thermal partons that constitutes the QGP. Although one implements Boltzmann transport driven by perturbative scattering matrix elements, temperature dependent running coupling and thermal mass are introduced, which are extracted from the lattice equation of state and 
effectively mimics certain non-perturbative effects and enhances the heavy-light parton interaction. Examples of quasi-particle models include PHSD [13], Catania-QPM [14] and AMPT [15].

At high momenta, perturbative approaches are widely applied to study the medium-induced gluon emission process from heavy quarks. Assuming heavy quarks interact with quasi-particles inside the QGP, one can evaluate the cross section of gluon emission with $2 \rightarrow 3$ matrices. Although results at the LO exist [16], their complication prevents an efficient implementation in numerical calculation. For this reason, the Gunion-Bertsch approximation [17] is adopted which is derived at the high energy limit and reproduces the exact result over a wide rapidity range. This approach is implemented in several models such as BAMPS [18], EPOSHQ [19] and Lido [20].

Without assuming the quasi-particle picture of the medium, one may consider inelastic scattering between a heavy quark with a general medium. The higher-twist formalism was first derived in deep inelastic scattering in large nucleus and then extended to hot nuclear matter [21, 22]. With collinear expansion $\left(\left\langle k_{\perp}^{2}\right\rangle \ll l_{\perp}^{2} \ll Q^{2}\right.$, where $k_{\perp}$ is the momentum transfer between the jet parton and the medium, $l_{\perp}$ is the transverse momentum of the emitted gluon, and $Q$ is the virtuality scale of the jet parton), one can obtain the medium-induced gluon spectra as a function of the path length of the jet parton inside the medium. The medium information is all absorbed in the jet transport parameter $\hat{q}=d\left\langle p_{\perp}^{2}\right\rangle / d t$, characterizing the transverse momentum broadening of the jet parton per unit time. This formalism has been adopted in several heavy quark studies such as the improved Langevin approach $[23,24]$ and LBT $[25,26]$. The higher-twist energy loss formalism has recently been improved in several directions. With a more delicate setup of the initial parton's transverse momentum that ensures gauge invariance, a larger heavy quark energy loss at low energy scale is observed compared to prior calculations [27]. In addition to the transverse momentum broadening induced radiation that is quantified by $\hat{q}$, longitudinal drag and diffusion induced radiation, quantified by $\hat{e}$ and $\hat{e}_{2}$, is also found important for slowly moving heavy quarks [28].

Alternative radiative energy loss formalisms are developed based on different assumptions. For instance, GLV [29, 30] is based on soft approximation where the fractional energy of the emitted gluon is assumed small compared to the parent parton; SCET [31] adopts both soft and collinear approximation. A detailed comparison of the path length dependence of the heavy quark radiative energy loss between these different formalisms has been explored in a recent EMMIRRTF report [32].

Heavy quark transport parameter $\hat{q}$ quantifies the strength of heavy quark interaction with the medium. First-principles calculation of $\hat{q}$ at finite momentum is still unavailable yet, thus it can only be extracted from model-to-data comparison. Consequently, the extracted value of $\hat{q}$ depends on the model one implements. A systematical investigation of this model dependence of the extracted $\hat{q}$ was initiated by the JET collaboration and has been recently reported in Ref. [33]. As shown in Fig. 1, results from 8 different models are compared on the same footing. To eliminate systematical uncertainties from the bulk medium and different hadronization models, a static medium with a fixed temperature at $250 \mathrm{MeV}$ is applied. All models are adjusted such that at the time of $t=3 \mathrm{fm}$, the nuclear modification factor $R_{\mathrm{AA}}$ of charm quarks equals 0.3 at $p_{\mathrm{T}}=15 \mathrm{GeV}$. With this common baseline, one observes the extracted values of $\hat{q}$ converge into three groups. Models including both elastic and inelastic energy loss converge to the smallest value; models with only elastic energy loss but based on perturbative calculations or the $T$-matrix method converge to the second group; and the remaining two quasi-particle models converge to the largest value. Due to the 

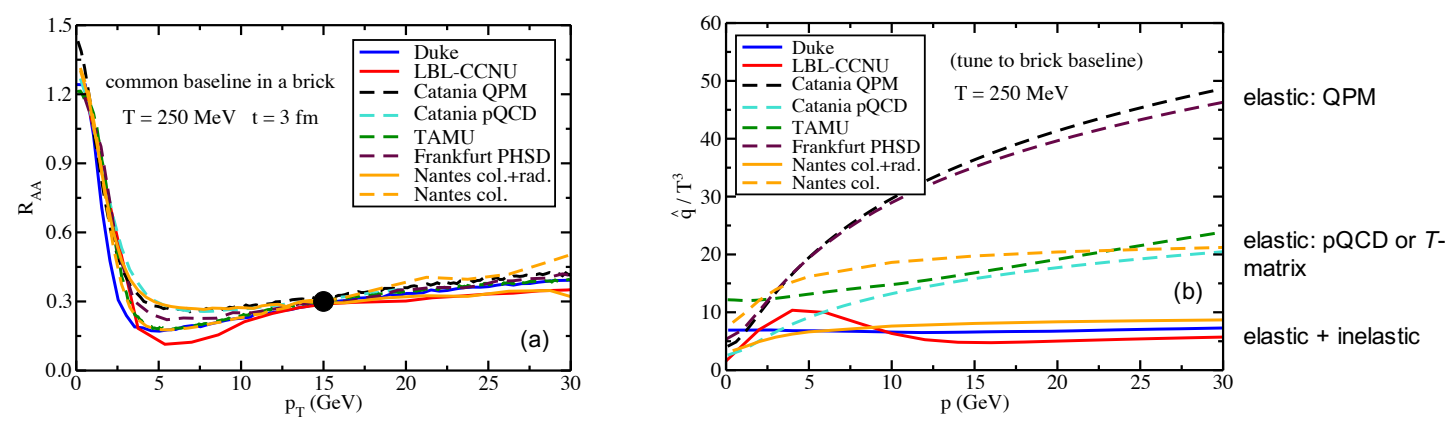

Figure 1: Model dependence of the extracted value of the heavy quark transport parameter $\hat{q}$.

larger thermal mass and Debye screening mass used in the quasi-particle models, they provide the largest transverse momentum broadening when the total amount of energy loss of heavy quarks is fixed. The difference presented here in the transverse momentum broadening is expected to be further distinguished by future experimental data on two-particle correlation functions of heavy quarks $[34,35]$.

Apart from energy loss, the hadronization process is also important to understand heavy quark observables, especially at medium $p_{\mathrm{T}}$. Hadronization is a non-perturbative process, and there is no first-principles calculation yet. Thus, modeling is inevitable. It is commonly agreed that high momentum heavy quarks tend to fragment directly into hadrons. One may apply a well-tuned fragmentation function to calculate the hadron spectra or use Pythia to simulate this process. On the other hand, it is more probable for low momentum heavy quarks to combine with thermal partons from the medium to form new hadrons. This is known as recombination, or coalescence mechanism.

There are two major recombination models. One is the instantaneous recombination model [36, 37], in which the probability for two partons to combine is given by the Wigner function that is defined as the wavefunction overlap between the two-constituent-quarks state and the final meson bound state. This approach is easy to be extended to a 3-body system for the baryon formation. But one disadvantage is it cannot rigorously guarantee the energy-momentum conservation in such a $2 \rightarrow 1$ process. Another model is known as resonant recombination [11], in which the coalescence probability is given by the product of the resonance lifetime window and the meson formation rate. The meson formation rate is further related to the cross section of the heavy-light quark resonance scattering. This method respects the energy-momentum conservation due to the existence of resonant states. In the EMMI-RRTF report [32], these two models are systematically compared, where one can observe the resonant recombination model usually gives smaller and softer $D$ meson spectra than the instantaneous recombination model.

The recombination mechanism is important to the medium $p_{\mathrm{T}}$ observables. It converts low $p_{\mathrm{T}}$ heavy quarks into medium $p_{\mathrm{T}} D$ mesons and thus enhances the $D$ meson $R_{\mathrm{AA}}$ at medium $p_{\mathrm{T}}$. It also adds the momentum space anisotropy of thermal light partons onto heavy quarks when $D$ mesons form and thus enhances the $D$ meson $v_{2}$. Additionally, recombination is essential in understanding the significant enhancement of the $\Lambda_{c} / D_{0}$ ratio in heavy-ion collisions compared to that in pp 


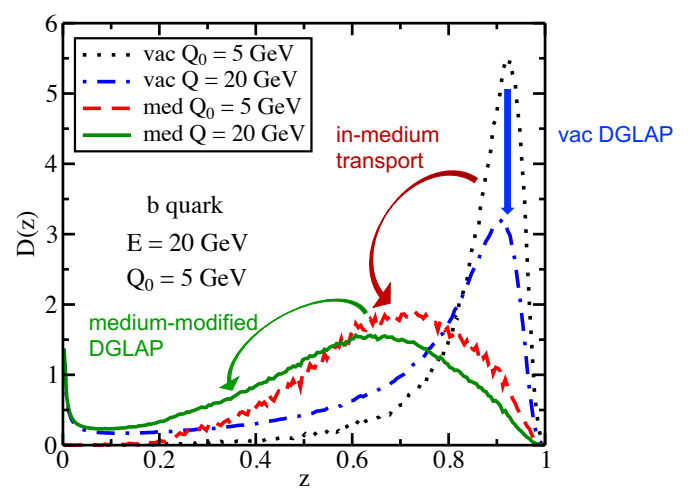

Figure 2: Multi-scale evolution of the heavy quark fragmentation function.

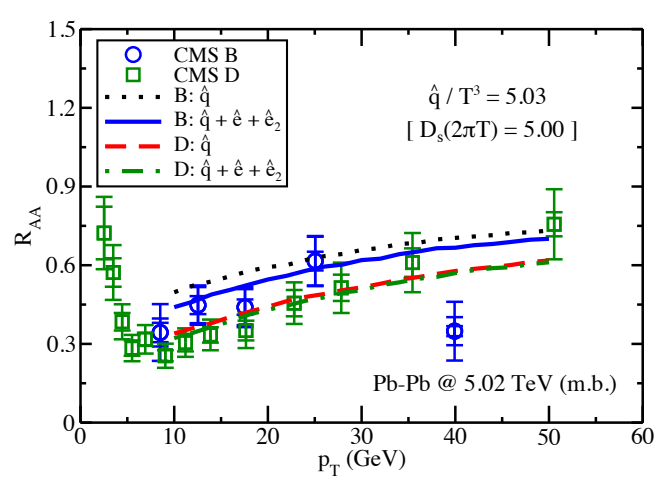

Figure 3: $R_{\mathrm{AA}}$ of $D$ and $B$ mesons from the multi-scale evolution approach.

collisions [2]. However, challenge still remains in current theoretical studies to precisely describe the $\Lambda_{c}$ versus $D$ chemistry observed in experimental data.

\section{Multi-scale approaches of heavy quark energy loss}

As discussed in the previous section, heavy quark interaction with the QGP at different scales are dominated by different physics. Therefore, a complete understanding of heavy quark observables requires multi-scale approaches that combine physics from different regions of phase space.

As a first example, the DGLAP-type of evolution and transport model have been unified into a single approach in Ref. [38]. When the virtuality scale of a heavy quark is much larger than the heavy meson mass, the heavy quark energy loss is dominated by rare scattering multiple emission, and its fragmentation function is typically described using the DGLAP equation. As an important input into the DGLAP equation, the medium-modified parton splitting function is taken from the higher-twist energy loss formalism [21]. At high virtuality, the medium-induced gluon radiation is dominated by the contribution from the transverse momentum broadening that is characterized by $\hat{q}$. Another crucial ingredient in the DGLAP equation is the input fragmentation function at a low virtuality scale $\left(Q_{0}\right)$. This is extracted from an on-shell transport model in this work. When the heavy quark virtuality is comparable to the heavy meson mass, its evolution is dominated by single scattering induced gluon emission. In this region, the DGLAP equation is no longer applicable. Instead, transport model with rate equations is a better choice. For slowly moving heavy quarks, the gluon emission rate is not only contributed by $\hat{q}$, but also contributed by longitudinal drag and diffusion, characterized by $\hat{e}$ and $\hat{e}_{2}$ [28].

Figure 2 illustrates the evolution of the $b$ quark fragmentation function through this multi-scale approach. One may start with a vacuum fragmentation function at a low virtuality scale $Q_{0}$ that is comparable with the $B$ meson mass ( $5 \mathrm{GeV}$ ), as shown by the black dotted curve. In vacuum, it can be evolved through the vacuum DGLAP equation into the vacuum fragmentation function at a high scale that equals to the heavy quark energy $(20 \mathrm{GeV})$, as shown by the blue dotted-dashed curve. This gives the $B$ meson spectra in pp collision after being convoluted with the initial $b$ quark spectra. On the other hand, inside a medium, the black curve can be first evolved into the red dashed curve through the in-medium transport model at low virtuality scale, and then further evolved into 


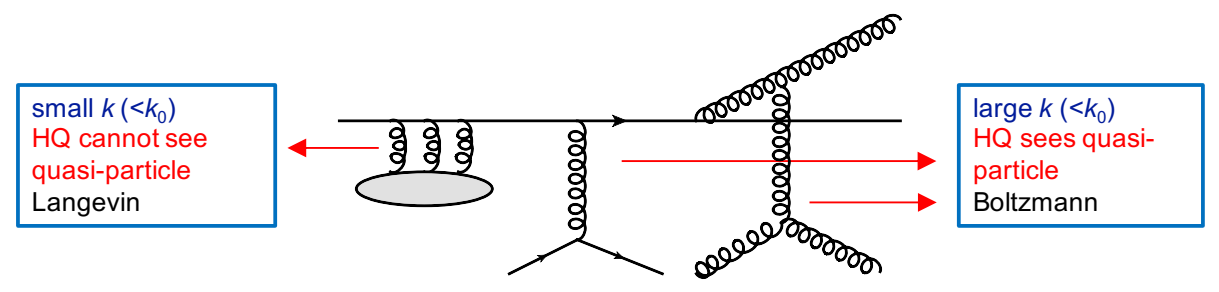

Figure 4: A multi-scale evolution that combines Langevin and Boltzmann transport.

the green solid curve through the medium-modified DGLAP equation to a high virtuality scale. This results in the $B$ meson spectra in AA collisions.

The nuclear modification factors of $D$ and $B$ mesons are presented in Fig. 3, compared between only including the transverse momentum broadening induced radiation $(\hat{q})$, and including the longitudinal drag and diffusion induced radiation ( $\hat{e}$ and $\left.\hat{e}_{2}\right)$. One observes that contributions from $\hat{e}$ and $\hat{e}_{2}$ at low virtuality scales are more important to $b$ quarks than to $c$ quarks because of the much larger mass of the former. These contributions are non-negligible at low $p_{\mathrm{T}}$ but naturally diminish as $p_{\mathrm{T}}$ increases. They narrow the difference of the $R_{\mathrm{AA}}$ between $D$ and $B$ mesons, which is favored by the experimental data.

Another example of multi-scale approach is combining Boltzmann and Langevin dynamics into a unified transport model. Boltzmann transport is a standard approach that describes scatterings between quasi-particles. In the limit of small momentum transfer, the Boltzmann equation can be simplified into the Langevin equation. However, as shown in Ref. [32], the Langevin results start to deviate from Boltzmann when the heavy quark mass $(M)$ is no longer much larger than the medium temperature $(T)$, i.e., $M / T \gg 1$ is not satisfied. On the other hand, the Langevin transport can be extended to heavy quark interaction with a non-quasi-particle medium where the Boltzmann transport is not applicable. Thus, neither Boltzmann nor Langevin alone is sufficient to describe the heavy quark interaction with the QGP. A novel approach, Lido, has been developed in Ref. [20] that combines these two models. As illustrated in Fig. 4, when the momentum exchange between a given heavy quark and the medium is smaller than a certain separation scale $\left(k<k_{0}\right)$, the heavy quark cannot "see" quasi-particles inside the medium, and thus its evolution is described as diffusion using the Langevin equation. To the contrary, when the momentum exchange is larger than the separation scale $\left(k>k_{0}\right)$, the heavy quark starts to "see" quasi-particles, thus its scatterings with the medium constituents are described using the Boltzmann equation.

To implement a multi-scale evolution, an advanced numerical framework is now available to the community from the JETSCAPE collaboration [39, 40]. It provides well-tuned initial conditions, hydrodynamical evolution, hadronization as well as several default jet energy loss modules. One may embed a new energy loss model into JETSCAPE and specify its applicable region in phase space. It will then work together with other default modules that complement the physics processes outside the user-defined region of phase space.

\section{Probing the QGP using heavy quarks}

Apart from improving theories and models for heavy quarks, it is of particular interest in utilizing heavy quarks to probe novel properties of the nuclear matter produced in energetic nuclear 
collisions. One long-standing question is whether heavy quarks can indeed reveal valuable information on the QGP evolution history, which is hard to observe from soft hadron spectra. A positive answer has been recently reported in a collaboration effort led by the Duke and Frankfurt groups [41]. The effects of different initial conditions of the bulk matter on heavy quark $v_{2}$ are explored within a common coupled heavy quark transport and fluid dynamics model. It is found that the geometric anisotropy (characterized by the $\varepsilon_{2}$ coefficient) of the hydrodynamic medium exhibit different time evolutions when different initial conditions are used. Although they lead to similar $\varepsilon_{2}$ values of the final state bulk medium, a clear difference in the final state heavy quark $v_{2}$ is observed, indicating the sensitivity of heavy quark observables to the QGP evolution history.

Last but not least, it has been suggested that heavy quarks are valuable probes of the initial state effects in high-energy nuclear collisions. In particular, the new puzzle in small system, where slight suppression [42] but large $v_{2}$ [43] are observed for $D$ mesons, urges us to explore what mechanism can cause small energy loss of heavy quarks but strong correlation between them. Although heavy quark interaction with the QGP has been extensively investigated, there are few studies on its interaction with a Glasma matter yet. It is possible that the correlation between multiple scatterings in the Glasma result in large $v_{2}$ of hard probes without causing significant energy loss. The study of heavy quark transport inside the Glasma has now been initiated in Refs. [44, 45]. To further confirm whether it is the Plasma or Glasma effect that contributes to the heavy quark $v_{2}$ in small systems, it is worth measuring the $v_{2}$ of direct photons in $\mathrm{p}-\mathrm{Pb}$ collisions. If photons do not exhibit large $v_{2}$, it is more likely that the Glasma effects on hard probes are crucial. On the other hand, if photons possess large $v_{2}$ as well, there must still be unknown properties of the Plasma matter. In addition to the Glasma effects, heavy quark interaction with the initial strong magnetic field in heavy-ion collisions has been investigated in Ref. [46], where the opposite sign of the direct flow $\left(v_{1}\right)$ at finite rapidity between $D$ and $\bar{D}$ mesons are predicted.

\section{Summary}

To conclude, this work has presented a brief overview of open heavy quark theories and models at different scales, including heavy quark diffusion at low $p_{\mathrm{T}}$, radiative energy loss at high $p_{\mathrm{T}}$ and hadronization at medium $p_{\mathrm{T}}$. Two multi-scale approaches have been introduced for heavy quark energy loss. One is the DGLAP + transport approach that combines theories at high and low virtuality scales; and the other is the Boltzmann + Langevin approach that combines transport models for high and low momentum exchange between heavy quarks and the QGP. In the end, recent theoretical progress has been discussed on utilizing heavy quarks to probe the medium properties, including probing the evolution history of the QGP, as well as probing the initial state Glasma and strong magnetic field effects in high-energy nuclear collisions.

\section{Acknowledgments}

This work is funded in part by the U.S. Department of Energy (DOE) under grant number DE-SC0013460 and in part by the National Science Foundation (NSF) under grant number ACI1550300 within the framework of the JETSCAPE collaboration. 


\section{References}

[1] STAR, L. Adamczyk et al., Phys. Rev. Lett. 118, 212301 (2017), arXiv:1701.06060.

[2] STAR, G. Xie, Nucl. Phys. A967, 928 (2017), arXiv:1704.04353.

[3] CMS, A. M. Sirunyan et al., (2018), arXiv:1810.11102.

[4] B. Svetitsky, Phys. Rev. D37, 2484 (1988).

[5] G. D. Moore and D. Teaney, Phys. Rev. C71, 064904 (2005), hep-ph/0412346.

[6] S. Caron-Huot and G. D. Moore, JHEP 0802, 081 (2008), arXiv:0801.2173.

[7] D. Banerjee, S. Datta, R. Gavai, and P. Majumdar, Phys. Rev. D85, 014510 (2012), arXiv:1109.5738.

[8] A. Francis, O. Kaczmarek, M. Laine, T. Neuhaus, and H. Ohno, Phys. Rev. D92, 116003 (2015), arXiv:1508.04543.

[9] H. T. Ding et al., Phys. Rev. D86, 014509 (2012), arXiv:1204.4945.

[10] H. van Hees, M. Mannarelli, V. Greco, and R. Rapp, Phys. Rev. Lett. 100, 192301 (2008), arXiv:0709.2884.

[11] M. He, R. J. Fries, and R. Rapp, Phys. Rev. C86, 014903 (2012), arXiv:1106.6006.

[12] S. Y. F. Liu and R. Rapp, Phys. Rev. C97, 034918 (2018), arXiv:1711.03282.

[13] T. Song et al., Phys. Rev. C92, 014910 (2015), arXiv:1503.03039.

[14] F. Scardina, S. K. Das, V. Minissale, S. Plumari, and V. Greco, Phys. Rev. C96, 044905 (2017), arXiv:1707.05452.

[15] H. Li, Z.-W. Lin, and F. Wang, (2018), arXiv:1804.02681.

[16] Z. Kunszt, E. Pietarinen, and E. Reya, Phys. Rev. D21, 733 (1980).

[17] O. Fochler, J. Uphoff, Z. Xu, and C. Greiner, Phys. Rev. D88, 014018 (2013), arXiv:1302.5250.

[18] J. Uphoff, O. Fochler, Z. Xu, and C. Greiner, (2014), arXiv:1408.2964.

[19] P. Gossiaux, J. Aichelin, T. Gousset, and V. Guiho, J. Phys. G37, 094019 (2010), arXiv:1001.4166.

[20] W. Ke, Y. Xu, and S. A. Bass, (2018), arXiv:1806.08848.

[21] B.-W. Zhang, E. Wang, and X.-N. Wang, Phys. Rev. Lett. 93, 072301 (2004), arXiv:nucl-th/0309040.

[22] A. Majumder, Phys. Rev. D85, 014023 (2012), arXiv:0912.2987.

[23] S. Cao, G.-Y. Qin, and S. A. Bass, Phys. Rev. C88, 044907 (2013), arXiv:1308.0617.

[24] S. Cao, G.-Y. Qin, and S. A. Bass, Phys. Rev. C92, 024907 (2015), arXiv:1505.01413.

[25] S. Cao, T. Luo, G.-Y. Qin, and X.-N. Wang, Phys. Rev. C94, 014909 (2016), arXiv:1605.06447.

[26] S. Cao, T. Luo, G.-Y. Qin, and X.-N. Wang, Phys. Lett. B777, 255 (2018), arXiv:1703.00822.

[27] Y.-L. Du, Y. He, X.-N. Wang, H. Xing, and H.-S. Zong, Phys. Rev. D98, 054015 (2018), arXiv:1807.06917.

[28] R. Abir and A. Majumder, Phys. Rev. C94, 054902 (2016), arXiv:1506.08648.

[29] M. Gyulassy, P. Levai, and I. Vitev, Nucl. Phys. B594, 371 (2001), arXiv:nucl-th/0006010.

[30] J. Xu, J. Liao, and M. Gyulassy, JHEP 02, 169 (2016), arXiv:1508.00552. 
[31] Z.-B. Kang, F. Ringer, and I. Vitev, JHEP 03, 146 (2017), arXiv:1610.02043.

[32] A. Beraudo et al., Nucl. Phys. A979, 21 (2018), arXiv:1803.03824.

[33] S. Cao et al., (2018), arXiv:1809.07894.

[34] S. Cao, G.-Y. Qin, and S. A. Bass, Nucl.Phys. A932, 38 (2014), arXiv:1404.1081.

[35] S. Cao, G.-Y. Qin, and S. A. Bass, Phys. Rev. C92, 054909 (2015), arXiv:1505.01869.

[36] Y. Oh, C. M. Ko, S. H. Lee, and S. Yasui, Phys. Rev. C79, 044905 (2009), arXiv:0901.1382.

[37] S. Li and C. Wang, Phys. Rev. C98, 034914 (2018), arXiv:1805.05807.

[38] S. Cao, A. Majumder, G.-Y. Qin, and C. Shen, (2017), arXiv:1711.09053.

[39] JETSCAPE, S. Cao et al., Phys. Rev. C96, 024909 (2017), arXiv:1705.00050.

[40] JETSCAPE, K. Kauder, (2018), arXiv:1807.09615.

[41] Y. Xu et al., (2018), arXiv:1809.10734.

[42] ALICE, J. Adam et al., Phys. Rev. C94, 054908 (2016), arXiv:1605.07569.

[43] CMS, Z. Shi, (2018), arXiv:1807.04362.

[44] S. Mrowczynski, Eur. Phys. J. A54, 43 (2018), arXiv:1706.03127.

[45] M. Ruggieri and S. K. Das, Phys. Rev. D98, 094024 (2018), arXiv:1805.09617.

[46] S. K. Das et al., Phys. Lett. B768, 260 (2017), arXiv:1608.02231. 